\title{
A multicenter open-label phase II trial to evaluate nivolumab and ipilimumab for 2nd line therapy in elderly patients with advanced esophageal squamous cell cancer (RAMONA)
}

Nadja M. Meindl-Beinker ${ }^{1 *}$ (D), Johannes Betge ${ }^{1}$, Tobias Gutting ${ }^{1}$, Elke Burgermeister ${ }^{1}$, Sebastian Belle ${ }^{1}$, Tianzuo Zhan ${ }^{1}$, Nadine Schulte ${ }^{1}$, Martin Maenz ${ }^{2}$, Matthias P. Ebert ${ }^{1}$ and Nicolai Haertel ${ }^{1}$

\begin{abstract}
Background: Advanced esophageal squamous cell cancer (ESCC) is frequently diagnosed in elderly patients. The impact of 2 nd line chemotherapy is poorly defined. Recent data demonstrated effectiveness of checkpoint inhibitors in different squamous cell carcinomas. Therefore, we assess combined nivolumab/ipilimumab as 2nd line therapy in elderly ESCC patients.

Methods: RAMONA is a multicenter open-label phase II trial. The primary objective is to demonstrate a significant survival benefit of nivolumab/ipilimumab in advanced ESCC compared to historical data of standard chemotherapy. Primary endpoint is therefore overall survival (OS). Major secondary objective is the evaluation of tolerability. Time to QoL deterioration will thus be determined as key secondary endpoint. Further secondary endpoints are tumor response, PFS and safety. We aim to recruit a total of $n=75$ subjects that have to be $>65$ years old. Eligibility is determined by the geriatric status (G8 screening and Deficit Accumulation Frailty Index (DAFI)). A safety assessment will be performed after a 3 cycle run-in phase of nivolumab (240 mg Q2W) to justify escalation for eligible patients to combined nivolumab (240 mg Q2W) and ipilimumab (1 mg/kg Q6W), while the other patients will remain on nivolumab only. RAMONA also includes translational research sub-studies to identify predictive biomarkers, including PD-1 and PD-L1 evaluation at different time points, establishment of organoid cultures and microbiome analyses for response prediction.

Discussion: The RAMONA trial aims to implement checkpoint inhibitors for elderly patients with advanced ESCC as second line therapy. Novel biomarkers for checkpoint-inhibitor response are analyzed in extensive translational sub-studies.
\end{abstract}

Trial registration: EudraCT Number: 2017-002056-86; NCT03416244, registered: 31.1.2018.

Keywords: Esophageal squamous cell cancer, Elderly, Comprehensive geriatric assessment, Checkpoint inhibitors, Personalized medicine, Geriatric oncology

\footnotetext{
* Correspondence: nadja.meindl-beinker@medma.uni-heidelberg.de

${ }^{1}$ Department of Medicine II, University Hospital Mannheim, Medical Faculty

Mannheim, Heidelberg University, Theodor-Kutzer-Ufer 1-3, 68167 Mannheim,

Germany

Full list of author information is available at the end of the article
}

(c) The Author(s). 2019 Open Access This article is distributed under the terms of the Creative Commons Attribution 4.0 International License (http://creativecommons.org/licenses/by/4.0/), which permits unrestricted use, distribution, and

reproduction in any medium, provided you give appropriate credit to the original author(s) and the source, provide a link to the Creative Commons license, and indicate if changes were made. The Creative Commons Public Domain Dedication waiver (http://creativecommons.org/publicdomain/zero/1.0/) applies to the data made available in this article, unless otherwise stated. 


\section{Background}

ESCC is the sixth leading cause of cancer-related death worldwide [1]. The disease is frequently diagnosed in advanced tumor stages and in elderly patients $[1,2]$.

Efficacy of chemotherapy in advanced ESCC is still poorly defined. While most patients undergo chemotherapy and/ or chemo-radiation in first line according to the CROSS protocol using Paclitaxel and Carboplatin, effectiveness of second-line chemotherapy is discouraging [3, 4]. However, very recently Kojima et al. reported that pembrolizumab significantly improved OS compared to chemotherapy (paclitaxel, docetaxel or irinotecan) in patients with advanced esophageal or esophagogastric junction carcinoma whose tumors express PD-L1 (Combined Positive Score [CPS] $\geq 10$, regardless of histology) (median 9.3 vs 6.7 mo; HR 0.69 ; $95 \%$ CI $0.52-0.93 ; P=0.0074)$. OS at 12 months was $43 \%$ vs $20 \%$, respectively. (KEYNOTE 181) [5].

Immunotherapy with antibodies against immune checkpoints like PD-1/PD-L1 represents a new treatment opportunity with relatively little side effects and first promising results in the treatment of squamous cell carcinoma patients [6-8]. With respect to esophageal cancer, preliminary results from an Asian study indicate efficacy of nivolumab [9]. From 64 heavily pre-treated patients, $11(17,95 \%$ CI 10-28) had an objective response and 16 (25, 95\% CI 16-37) demonstrated stable disease. The median overall survival was 10.8 months (IQR 4.9-14.3) in this trial population (unselected for PD-L1 expression status). Long-term survival was also improved by pembrolizumab as described by Doi et al. [10].

Furthermore, the CheckMate 012 trial demonstrated that overall response rates could be doubled when PD-1 inhibitor nivolumab was combined with CTLA-4-inhibitor ipilimumab in advanced NSCLC patients [11]. In this trial, grade 3 and 4 adverse events were reported to occur in $33 \%$ of the patients treated with the combination therapy (nivolumab $3 \mathrm{mg} / \mathrm{kg} \mathrm{Q} 2 \mathrm{~W}$ and ipilimumab $1 \mathrm{mg} / \mathrm{kg}$ Q6W). In the checkmate 032 in turn (nivolumab $3 \mathrm{mg} / \mathrm{kg}$ Q2W and ipilimumab $1 \mathrm{mg} / \mathrm{kg} \mathrm{Q} 3 \mathrm{~W}$ ), treatment related adverse events of grade 3 and 4 were only slightly enhanced when compared to nivolumab monotherapy (13\% vs. 19\%) [12].

There is an increasing need for improved treatment strategies for elderly ESCC patients. These strategies have to acknowledge the challenges of functional limitations and comorbidities in this increasing population. With increasing age, elderly patients develop chronic diseases and different comorbidities that may affect person's capabilities, functional reserve and life expectancy [13]. However, assessment of these characteristics in the elderly population is time-consuming, therefore new assessment and screening tools are being developed. The poor knowledge of the role of chemotherapy and immunotherapy in these individuals, due to lack of enrolment of these patients in clinical trials, demands for novel concepts of clinical trials specifically designed for elderly patients.

With the RAMONA trial, we aim to address this high medical need by assessing nivolumab and ipilimumab in combination as second-line therapy of advanced ESCC in the elderly population. Patient eligibility will be assessed by geriatric screening tools in this trial. Moreover, we aim to establish novel biomarkers for checkpoint inhibition by extensive translational sub-studies.

\section{Methods/design Study design}

RAMONA is a multicenter open-label phase II trial conducted in 34 centers in Germany (for overviews please refer to Additional file 1: Table S1). Key inclusion criteria are

- age $\geq 65$ years at time of recruitment

- histologically approved diagnosis of advanced ESCC

- progression after front line treatment (including chemo-radiation with carboplatin/paclitaxel or others)

A screening phase is used to determine the eligibility of a patient and may last up to 4 weeks before initiation of treatment. Eligibility and the geriatric status of potential patients will be assessed using the G8 screening tool and the Deficit Accumulation Frailty Index (DAFI). Patients are recruited independent of their PD-1 or PD-L1 expression status.

The dosing rationale for the study is based on Kudo et al. [9] and Hellmann et al. [11]. The treatment phase begins with 2nd-line nivolumab monotherapy safety run-in (240 mg fixed dose Q2W). Based on reports by Kähler et al., $[14,15]$ a safety assessment will be performed after 3 cycles of nivolumab monotherapy (6 weeks), during which the investigator will decide if a patient is eligible to receive nivolumab/ipilimumab combination therapy (Nivo $240 \mathrm{mg}$ fixed dose Q2W; ipilimumab $1 \mathrm{mg} / \mathrm{kg}$ Q6W; arm A) or continues nivolumab monotherapy (240 mg fixed dose Q2W; arm B). This safety assessment was added due to the substantial comorbidities in most ESCC patients which may increase adverse events as compared to patients with other carcinomas and which might e.g. counteract treatment escalation. Only those patients with toxicities grade $\leq 2$ will be escalated. In both arms, treatment continues until progressive disease or intolerable toxicity or withdrawal of consent or death. After another 3 cycles of treatment in both arms (approx.12 weeks from first dose) a restaging examination by radiologic imaging will be performed, which is repeated every four treatment cycles thereafter. 
Treatment within the context of the study is limited to 2 years.

Samples for translational research are gathered at different time points, i.e. screening/baseline (no therapy), safety assessment (after 3 cycles of nivolumab monotherapy) and restaging (after 3 cycles in treatment arm A or arm B). The study design is illustrated in Fig. 1.

\section{Study objectives}

The primary objective of this trial is to demonstrate a significant survival benefit of the combination therapy with nivolumab/ipilimumab treatment in advanced ESCC compared to historical data of standard chemotherapy regimens [4].

Secondary objectives:

Tolerability of nivolumab as single agent and in combination with ipilimumab will be investigated in terms of QoL. Hence, a key secondary endpoint 'time to QoL deterioration' will be implemented.

Further secondary objectives are the assessment of additional efficacy and safety parameters of an intensified immunotherapy regimen and the assessment and exploration of the predictive value of structured geriatric assessments for treatment-emergent toxicities and treatment discontinuation.

\section{Measurements}

For patient screening, the G8 screening tool and the Deficit Accumulation Frailty Index (DAFI) will be used. Patients with G8 score $>14$ will be stratified as non-frail and can be directly included into to study (GoGo). If G8 scores $\leq 14$ (frail), the DAFI questionnaire needs to be performed. DAFI-indices between 0.2 and 0.35 qualify patients as SlowGo enabling the PI to decide whether a patient is eligible or cannot participate. DAFI index $\geq 0.35$ is defined as exclusion criterion.

In terms of safety, the screening includes an intensified assessment of cardiac co-morbidities using echocardiogram and measurement of cardiac enzymes (e.g. troponin-I). QoL will be assessed by the EORTC questionnaires QLQ-C30 and ELD14.

\section{Safety assessment}

Procedures to be conducted during the treatment phase of the study include a safety assessment after 3 weeks (3 cycles) of nivolumab monotherapy. A study subject is eligible to escalate to nivolumab/ipilimumab combination therapy if I) the study subject has sufficient cardiac

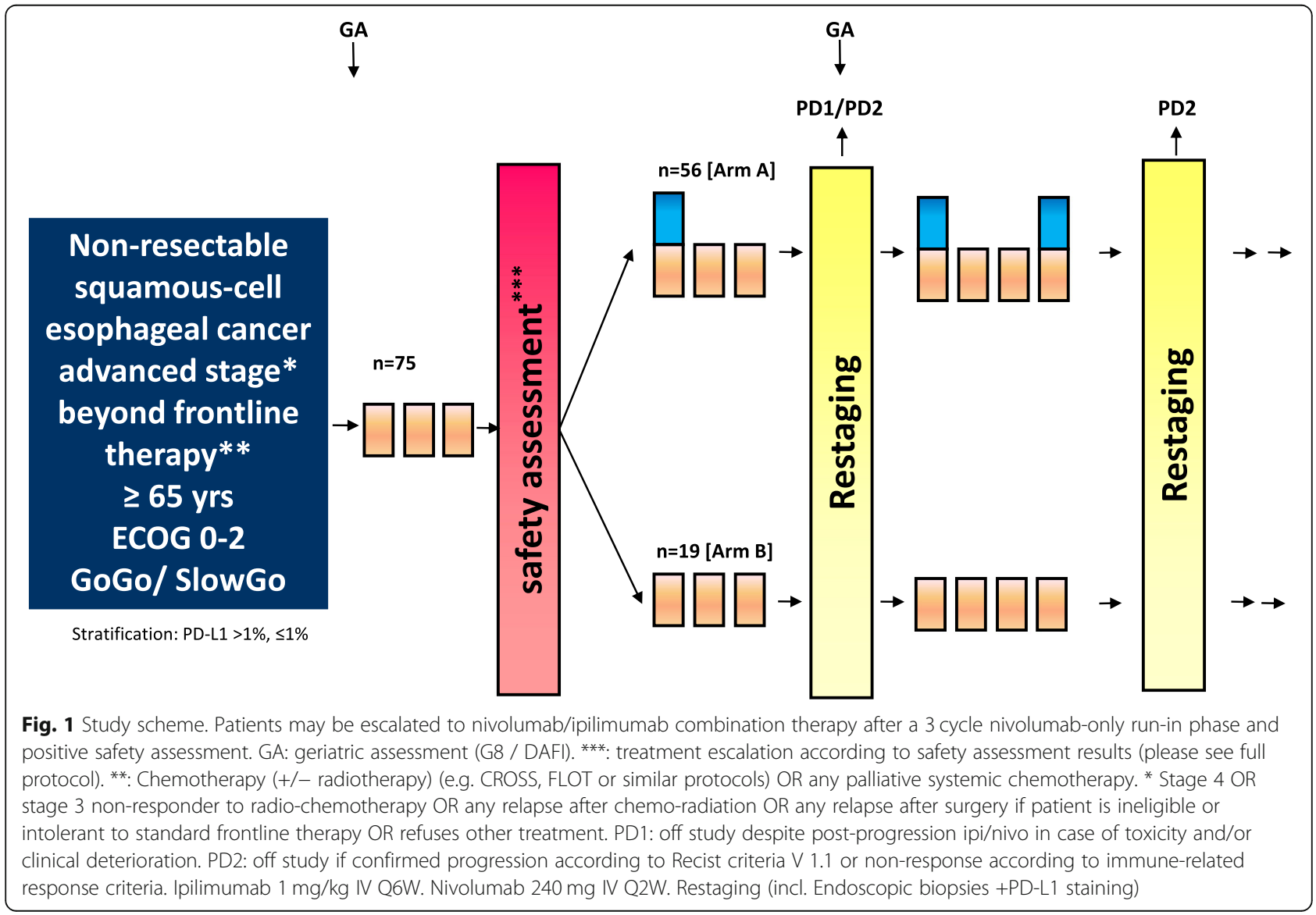


functional reserve determined by cardiac echo (less than $10 \%$ decline in ejection fraction compared to baseline assessment), II) no clinically significant abnormal troponin or ECG and III) the study subject is potentially benefitting from treatment escalation according to the judgment of the investigator.

\section{End of treatment}

All subjects will be followed for survival until the end of the study, regardless of further treatments, or until the sponsor ends the study (follow-up extension phase).

The following procedures will be performed during follow-up every 6 weeks for the first year of follow-up, and every 8-12 weeks thereafter:

- assessment of survival status

- recording of all anti-cancer treatments

- recording of all AEs and SAEs for 100 days after last dosing

\section{Primary and secondary end points \\ Primary endpoint}

- Overall survival

\section{Secondary endpoints}

Key secondary endpoint:

- Time to QoL deterioration defined as a loss of $\geq 10$ points in the EORTC QLQ-C30 compared to base-line

Additional secondary endpoints:

- PFS

- ORR according to RECIST 1.1 and immune related response criteria (modified RECIST)

- Duration of Response (DOR)

- Duration of treatment

- cumulative dose intensity

- QoL (EORTC QLQC30 and ELD14)

- AEs/SAEs, toxicities according to CTC criteria v4.0

- Geriatric assessments:

- Evaluation of the predictive value of the GA containing tests (DAFI, G8-Questionaire etc.) for the occurrence of $\geq$ grade 3 toxicities

- Predictive value of the assessed geriatric tests for treatment discontinuation

- Translational research at different time points (screening, safety assessment and first restaging)

- PD-1-PD-L1 expression in tumor tissue before and during treatment

- predictive biomarkers in tumor tissue (pre-treatment and re-biopsies) and blood
- establishment of organoid cultures from tumor tissue specimens

- Response prediction by microbiome assessment

\section{Study setting}

Patients will be recruited at 34 selected study sites in Germany including academic hospitals, community clinics and practitioner to achieve adequate participant enrolment. A list of study sites can be obtained from the corresponding author of this manuscript. Protocol modifications will be communicated to relevant parties upon ethics approval.

\section{Data collection}

Data for this study will be recorded via eCRF by the site from the source documents according to standard operational procedures. Data are reviewed and checked for omissions, apparent errors, and values requiring further clarifications using computerized (automatic) and/or manual procedures. Accurate and reliable data collection will be assured by verification and cross-check of the eCRF against the investigator's records by the study monitor. Data will be recorded and reported until the last subject will have completed the trial. Informed consent or assent forms including additional informed consent/assent forms for participation in the RAMONA translational research program will remain at the respective study sites. Confidentiality of personal information is protected by adherence to the European GDPR.

\section{Statistical analysis and sample size}

It is hypothesized that nivolumab and ipilimumab will increase overall survival. It is assumed that an immunotherapy approach consisting of a nivolumab monotherapy in conjunction with a safety guided treatment escalation to a nivolumab/ipilimumab combination regimen increases the 1-year overall survival rate by a margin of $13 \%$ compared to historical control for standard chemotherapy (i.e. nivolumab monotherapy followed by a conditional nivolumab + ipilimumab therapy 1-yr-OS $=30 \%$ vs CTx-control 1 -yr-OS $=17 \%$ ) [4]. Based on these assumptions, and an exponential shape of the survival curve, a one-sided, one-sample log rank test calculated from a sample of 69 subjects achieves $90.3 \%$ power at an alpha $=0.05$ one-sided significance level to detect a proportion surviving of 0.3 in the experimental group when the proportion surviving in the historic control group is 0.17 . These proportions surviving are for a period of 12 month (1-year-OS rate). Subjects are accrued for a period of 12 month. Follow-up continues for a period of 24 month after the last subject is added. The probability that a subject experiences an event during the study is 0.9477 . The expected number of 
events during the study is 65 . To compensate for uninformative drop-outs a total of $N=75$ subjects need to be recruited. All patients, both on monotherapy and on combination therapy, are subject to statistical ITT analysis.

\section{Discussion}

In the CROSS trial, van Hagen et al. demonstrated that first-line neoadjuvant chemoradiation using carboplatin and paclitaxel is a highly effective regimen for locally advanced ESCC, leading to patients' overall survival of more than 50 months in single cases (median DOR of 15 months) [3]. This approach is also considered effective as definite chemoradiotherapy for advanced ESCC patients who are not candidates for subsequent surgery [16] and therefore commonly used in Germany. In these patients, second line treatment with paclitaxel is not an option. However, treatment options after chemoradiation with platin derivatives and taxanes for patients with recurrent cancers are limited. Thallinger and coworkers convincingly demonstrated that 2nd line therapy for these patients is un-standardized [4]. Clinical trials focusing on 2nd line chemotherapy for ESCC patients showed frustrating response rates, enhanced toxicity rates and poor overall survival, mostly limited to 6-8 months [4]. Furthermore, majority of ESCC patients are elder persons [17] with significant co-morbidities which in the clinical practice typically renders these patients ineligible for poly-chemotherapy.

To address this issue, the aim of the RAMONA trial is to evaluate a promising new second line therapy option for elderly ESCC patients. Nivolumab as immunotherapeutical agent blocks interaction of PD-1 molecules expressed by tumor-specific T-cells with its ligand PD-L1 on tumor cells, as reviewed in [18]. PD-L1 expression seems to be significantly increased in ESCC with age and is associated with poor prognosis [19]. Pooled analysis of CheckMate 017 and checkmate 057 studies demonstrated, that higher PD-L1 expression levels were associated with greater OS benefit with nivolumab (HR, $0.42 ; 95 \% \mathrm{CI}, 0.28$ to 0.63 ) in patients with $250 \%$ PD-L1 expression, but a benefit was still observed in patients with $<1 \%$ PD-L1 expression (HR, 0.78; 95\% CI, 0.61 to 0.99) [20]. However, here effects seem to more prominent in non-squamous NSCLC. Antonia and colleagues very recently presented new data hinting towards improved ORR in correlation with PDL-1 expression levels upon nivolumab/ipilimumab combination therapy as compared to nivolumab monotherapy (Chicago Multidisciplinary Symposium in Thoracic Oncology, 2016, [11]). Despite further promising results indicating a correlation of good ORR with expression of PD-L1 in lung cancer (PD1 expression $\geq 1 \%$ ORR $57 \%$ vs. $28 \%$ ) $[11,21]$, the predictive value of PD-1/PD-L1 expression for nivolumab-based therapies is not fully understood in most tumor entities. Therefore, we implemented a translational part into the trial monitoring PD-1/PD-L1 expression rates during the course of treatment.

Checkpoint-inhibitor treatments combine several advantages, which are especially interesting with regard to ESCC treatment approaches. Firstly, promising overall survival has been reported in squamous cell carcinoma patients of different entities, pointing towards a rationale to test efficacy also in $\operatorname{ESCC}[6,11$, 22-24]. Secondly, antitumor activity of checkpoint inhibitors was shown when used after radiation-based treatments, which is commonly also performed in ESCC treatment. For instance, in a randomized phase III trial including stage II NSCLC patients (i.e. patient that undergo surgery with curative intent), Antonia and coworkers demonstrated that PFS was increased by durvalumab treatment after chemotherapy/radiotherapy compared to chemotherapy/radiotherapy alone [25]. In line, NSCLC patients treated with pembrolizumab showed longer PFS (HR 0.56 [95\% CI $0.34-0.91], \quad p=0.019$ ) and increased OS (HR 0.58 [95\% CI 0.36-0.94], $p=0.026)$ if they had received any radiotherapy as compared to non-radiated patients [26]. An association of radiotherapy with improved rates of index lesion response in $68 \%$ of melanoma patients treated with ipilimumab [27] further underline possible synergistic effects of radiation and immunotherapy on local on distant tumor control as reviewed in [28].

Kudo et al. reported that even for heavily pretreated ESCC patients, immunotherapy with nivolumab alone prolonged overall survival of Asian patients by median 10.8 months (95\% CI 7.4-13.3) [9]. Additionally, the risk profile of ESCC patients [1,29-32] in part overlaps with that of NSCLC patients who are often heavy smokers. In line, available data reveals that especially heavy smokers seem to profit from immunotherapy (Objective response: current and former smokers vs never-smokers (46\% [30 of 65 ] vs $27 \%$ [ 3 of 11]) [11].

ESCC is most frequently diagnosed between 65 and 74 years [17], defining the patient cohort as mostly elderly with per se accumulated co-morbidities, impaired pharmacokinetics and a potentially declined functional reserve [13]. To objectify the functional reserve of the patients and its impact on treatment outcome, a comprehensive geriatric assessment (CGA) is recommended [33]. However, CGA is time consuming and rarely feasible in clinical practice. Thus, we decided to assess the vulnerability of our study subjects by the G8 questionnaire and implement the more complex DAFI 
assessment only for patients below G8 cut-off $(\leq 14$ points). The DAFI is a condensed, validated CGA with 51 items lasting approximately $30 \mathrm{~min}$ of which $>80 \%$ can be self-reported by the patient [34]. Based on Cohen et al., we estimated that $\sim 40 \%$ of the patients will belong to a pre-frail group with DAFI indices between 0.2 and 0.35 . These patients can enter the RAMONA study upon investigator's decision as immune-oncological treatments are known to be well-tolerated [11]. Even for combined nivolumab/ipilimumab therapy, grade $\geq 3$ toxicities are reported to occur in only $33 \%$ in NSCLC [11]. As the CheckMate Trial 214 revealed grade 3 and 4 toxicities in up to $46 \%$ of the patients with advanced renal cell carcinoma treated with nivolumab and ipilimumab [35], we consider the RAMONA dosage treatment scheme with longer intervals of ipilimumab administration to provide better tolerability (CheckMate 214 ipilimumab Q3W as compared to Q6W in our study).

However, to ensure that patients are eligible for nivolumab/ipilimumab combination therapy in the RAMONA trial, a safety assessment is performed after a 3 week run-in phase of nivolumab alone. Only those patients with toxicities grade $\leq 2$ will be escalated.

Taken together, we used the findings discussed above to formulate the rationale of the RAMONA study and to transfer current knowledge on immunotherapy to the fragile cohort of elderly Caucasian ESCC patients. Overall survival was selected as primary endpoint. In line, impressive long term responses of immunotherapy (at least with pembrolizumab) of up to 50 months (median time to initial response of 4 months (range, 2 to 8 months); median DOR of 15 months (range, 6 to $\geq 26$ months) are reported, even after end of treatment [10]. Treatment adherence is often negatively impacted by quality of life (QoL) deterioration in cancer patients. Therefore, adequate preservation of QoL is an important clinical goal in in elderly patients with recurrent ESCC. Thus, 'Time to QoL Deterioration' was included as a key secondary endpoint in this trial.

\section{Conclusion}

In conclusion, RAMONA is the first trial to evaluate checkpoint inhibitor based immunotherapy as a novel treatment opportunity in elderly ESCC patients. Based on a recruitment period of 12 months, first results are expected end of 2019.

\section{Trial status}

RAMONA is an ongoing study. Recruitment commenced in April 2018 and the study is at the moment recruiting patients. For a status update please refer to https://clinicaltrials.gov/ct2/show/NCT03416244.

\section{Additional file}

Additional file 1: Table S1. RAMONA. Title of data: RAMONA synopsis. Description of data: synopsis of the RAMONA trial. (DOCX $37 \mathrm{~kb}$ )

\section{Abbreviations}

AEs/SAEs: Adverse events, serious adverse events; CGA: Comprehensive geriatric assessments; $\mathrm{Cl}$ : Confidence interval; CTC : Common toxicity criteria; CTLA-4: Cytotoxic T-lymphocyte-associated Protein 4; DAFI: Deficit accumulation frailty index; DOR : Duration of Response; ECOG: Eastern Cooperative Oncology Group; ESCC : Esophageal squamous cell cancer; HR: Hazard ratio; ORR: Objective response rate; OS: Overall survival; PD-1, PDL1: Programmed cell death protein 1, Programmed death-ligand 1; PFS: Progression-free survival; QLQ-C30: Quality of life questionnaire C30; QLQ-ELD14: Quality of life questionnaire ELD14; QoL: Quality of life; RECIST : Response Evaluation Criteria In Solid Tumors

\section{Acknowledgements}

None.

\section{Funding}

This investigator-initiated trial is sponsored by AlO-Studien $\mathrm{gGmbH}$ (Berlin, Germany) with funding from BMS. BMS had no role in the study design, and has no role in data collection, management, data analysis and interpretation, or decision to submit results for presentation or publication. Research in the Lab of ME is supported by grants from the State of Baden-Württemberg for "Center of Geriatric Biology and Oncology (ZOBEL) - Perspektivförderung" and "Biology of Frailty - Sonderlinie Medizin". JB is supported by the Translational Physician Scientist (TraPS) Program of the Medical Faculty Mannheim and the State of Baden-Württemberg. We acknowledge financial support by Deutsche Forschungsgemeinschaft within the funding programme Open Access Publishing, by the Baden-Württemberg Ministry of Science, Research and Arts and by Ruprecht-Karls-Universität Heidelberg.

\section{Availability of data and materials}

This study protocol has been prospectively registered on ClinicalTrials.gov registry no. NCT03416244. Data sharing is not applicable to this article as this is the report of a study protocol.

\section{Authors' contributions}

$\mathrm{NH}$ and MPE designed the study. MPE is principal investigator of the RAMONA trial, NH is substitute principle investigator. JB, SB, TZ and NS are members of the study team and made substantial contributions to conception and design. $\mathrm{NMB}, \mathrm{EB}$ and TG organize the translational research branch of the trial. MM is representative of the AIO Studien $\mathrm{gGmbH}$ (KunoFischer-Str. 8, 14,057 Berlin, Germany, info@aio-studien-ggmbh.de) and substantially contributed to conception and design. The non-profit AlOStudien $\mathrm{gGmbH}$ is sponsor of the study and responsible for study management, logistics and monitoring and counseled in study design. NMB and $\mathrm{NH}$ drafted the manuscript. All authors read and approved the final manuscript.

\section{Ethics approval and consent to participate}

The study was approved by the lead ethics committee of the study, Ethics Committee II at Medical Faculty Mannheim, Heidelberg University, Mannheim, Germany [2017-004F-MA] providing approval for all 34 study sites. The approval was permitted in agreement with local ethics commissions of the participating study sites. Written informed consent for participation is obtained from all participants.

\section{Consent for publication}

Not applicable.

\section{Competing interests}

This investigator-initiated trial is sponsored by the non-profit AlO-Studien $\mathrm{gGmbH}$ and received funding from Bristol-Myers Squibb (BMS) $\mathrm{GmbH} \& \mathrm{Co}$. KGaA, NY, USA under study contract.

BMS had no role in the study design, and has no role in data collection, management, data analysis and interpretation, or decision to submit results for presentation or publication. 
$\mathrm{NH}$ participated at advisory boards organized by the BMS (presentation incl.), MPE served on advisory board meetings of BMS.

The authors report no further conflicts of interest.

\section{Publisher's Note}

Springer Nature remains neutral with regard to jurisdictional claims in published maps and institutional affiliations.

\section{Author details}

${ }^{1}$ Department of Medicine II, University Hospital Mannheim, Medical Faculty Mannheim, Heidelberg University, Theodor-Kutzer-Ufer 1-3, 68167 Mannheim, Germany. ${ }^{2}$ AlO-Studien gGmbH, Berlin, Germany.

Received: 18 October 2018 Accepted: 7 March 2019

Published online: 14 March 2019

\section{References}

1. Abnet CC, Arnold M, Wei WQ. Epidemiology of esophageal squamous cell carcinoma. Gastroenterology. 2018;154(2):360-73.

2. Okada E, Ukawa S, Nakamura K, Hirata M, Nagai A, Matsuda K, Ninomiya T, Kiyohara Y, Muto K, Kamatani Y, et al. Demographic and lifestyle factors and survival among patients with esophageal and gastric cancer: the biobank Japan project. J Epidemiol. 2017;27(3S):S29-35.

3. van Hagen $P$, Hulshof MC, van Lanschot JJ, Steyerberg EW, van Berge Henegouwen MI, Wijnhoven BP, Richel DJ, Nieuwenhuijzen GA, Hospers GA, Bonenkamp JJ, et al. Preoperative chemoradiotherapy for esophageal or junctional cancer. N Engl J Med. 2012;366(22):2074-84.

4. Thallinger CM, Raderer M, Hejna M. Esophageal cancer: a critical evaluation of systemic second-line therapy. J Clin Oncol. 2011;29(35):4709-14.

5. Takashi Kojima KM, Francois E, Hsu C-H, Moriwaki T, Kim S-B, Lee S-H, Bennouna J, Kato K, Lin S, Qin S-Q, Ferreira P, Doi T, Adenis A, Enzinger PC, Shah MA, Wang R, Bhagia P, Peter Kang S, Metges J-P. Pembrolizumab versus chemotherapy as second-line therapy for advanced esophagea cancer: phase III KEYNOTE-181 study. J Clin Oncol. 2019;37(suppl 4):abstr 2.

6. Ferris RL, Blumenschein G, Jr., Fayette J, Guigay J, Colevas AD, Licitra L, Harrington K, Kasper S, Vokes EE, Even C et al: Nivolumab for recurrent squamous-cell carcinoma of the head and neck. N Engl J Med 2016, 375(19):1856-1867.

7. Brahmer J, Reckamp KL, Baas P, Crino L, Eberhardt WE, Poddubskaya E, Antonia S, Pluzanski A, Vokes EE, Holgado E, et al. Nivolumab versus docetaxel in advanced squamous-cell non-small-cell lung Cancer. N Engl J Med. 2015;373(2):123-35.

8. Seiwert TY, Burtness B, Mehra R, Weiss J, Berger R, Eder JP, Heath K, McClanahan T, Lunceford J, Gause C, et al. Safety and clinical activity of pembrolizumab for treatment of recurrent or metastatic squamous cell carcinoma of the head and neck (KEYNOTE-012): an open-label, multicentre, phase 1b trial. Lancet Oncol. 2016;17(7):956-65.

9. Kudo T, Hamamoto Y, Kato K, Ura T, Kojima T, Tsushima T, Hironaka S, Hara $H$, Satoh T, Iwasa S, et al. Nivolumab treatment for oesophageal squamouscell carcinoma: an open-label, multicentre, phase 2 trial. Lancet Oncol. 2017; 18(5):631-9.

10. Doi T, Piha-Paul SA, Jalal SI, Saraf S, Lunceford J, Koshiji M, Bennouna J. Safety and antitumor activity of the anti-programmed Death-1 antibody Pembrolizumab in patients with advanced esophageal carcinoma. J Clin Oncol. 2018;36(1):61-7.

11. Hellmann MD, Rizvi NA, Goldman JW, Gettinger SN, Borghaei H, Brahmer JR, Ready NE, Gerber DE, Chow LQ, Juergens RA, et al. Nivolumab plus ipilimumab as first-line treatment for advanced non-small-cell lung cancer (CheckMate 012): results of an open-label, phase 1, multicohort study. Lancet Oncol. 2017;18(1):31-41.

12. Antonia SJ, Lopez-Martin JA, Bendell J, Ott PA, Taylor M, Eder JP, Jager D, Pietanza MC, Le DT, de Braud F, et al. Nivolumab alone and nivolumab plus ipilimumab in recurrent small-cell lung cancer (CheckMate 032): a multicentre, open-label, phase 1/2 trial. Lancet Oncol. 2016;17(7):883-95.

13. Balducci L, Extermann M. Management of cancer in the older person: a practical approach. Oncologist. 2000;5(3):224-37.

14. Kahler KC, Hassel JC, Heinzerling L, Loquai C, Mossner R, Ugurel S, Zimmer L, Gutzmer R. Cutaneous side effects Committee of the Work Group Dermatological O: management of side effects of immune checkpoint blockade by anti-CTLA-4 and anti-PD-1 antibodies in metastatic melanoma. J Dtsch Dermatol Ges. 2016;14(7):662-81.
15. Kahler KC, Hauschild A. Treatment and side effect management of CTLA-4 antibody therapy in metastatic melanoma. J Dtsch Dermatol Ges. 2011;9(4):277-86

16. Noronha V, Prabhash K, Joshi A, Patil VM, Talole S, Nakti D, Sahu A, Shah S, Ghosh-Laskar S, Patil PS, et al. Clinical outcome in definitive concurrent Chemoradiation with weekly paclitaxel and carboplatin for locally advanced esophageal and junctional Cancer. Oncol Res. 2016;23(4):183-95.

17. Surveillance, Epidemiology, and End Results (SEER) Program Populations (1975-2015), National Cancer Institute, DCCPS, Surveillance Research Program, Cancer Statistics Branch. Available: https://seer.cancer.gov/ statfacts/html/esoph.html. Accessed 2018 August.

18. Drake CG, Lipson EJ, Brahmer JR. Breathing new life into immunotherapy: review of melanoma, lung and kidney cancer. Nat Rev Clin Oncol. 2014; 11(1):24-37.

19. Chen MF, Chen PT, Chen WC, Lu MS, Lin PY, Lee KD. The role of PD-L1 in the radiation response and prognosis for esophageal squamous cell carcinoma related to IL-6 and T-cell immunosuppression. Oncotarget. 2016;7(7):7913-24.

20. Horn L, Spigel DR, Vokes EE, Holgado E, Ready N, Steins M, Poddubskaya E, Borghaei H, Felip E, Paz-Ares L, et al. Nivolumab versus docetaxel in previously treated patients with advanced non-small-cell lung Cancer: twoyear outcomes from two randomized, open-label, phase III trials (CheckMate 017 and CheckMate 057). J Clin Oncol. 2017;35(35):3924-33.

21. Passiglia F, Bronte G, Bazan V, Natoli C, Rizzo S, Galvano A, Listi A, Cicero G, Rolfo C, Santini D, et al. PD-L1 expression as predictive biomarker in patients with NSCLC: a pooled analysis. Oncotarget. 2016;7(15):19738-47.

22. Chow LQM, Haddad R, Gupta S, Mahipal A, Mehra R, Tahara M, Berger R, Eder JP, Burtness B, Lee SH, et al. Antitumor activity of Pembrolizumab in biomarker-unselected patients with recurrent and/or metastatic head and neck squamous cell carcinoma: results from the phase lb KEYNOTE-012 expansion cohort. J Clin Oncol. 2016;34(32):3838-45.

23. Morris VK, Salem ME, Nimeiri H, lqbal S, Singh P, Ciombor K, Polite B, Deming D, Chan E, Wade JL, et al. Nivolumab for previously treated unresectable metastatic anal cancer (NC19673): a multicentre, single-arm, phase 2 study. Lancet Oncol. 2017;18(4):446-53.

24. Kang YK, Boku N, Satoh T, Ryu MH, Chao Y, Kato K, Chung HC, Chen JS, Muro K, Kang WK, et al. Nivolumab in patients with advanced gastric or gastro-oesophageal junction cancer refractory to, or intolerant of, at least two previous chemotherapy regimens (ONO-4538-12, ATTRACTION-2): a randomised, double-blind, placebo-controlled, phase 3 trial. Lancet. 2017; 390(10111):2461-71.

25. Antonia SJ, Villegas A, Daniel D, Vicente D, Murakami S, Hui R, Yokoi T, Chiappori A, Lee KH, de Wit M, et al. Durvalumab after Chemoradiotherapy in stage III non-small-cell lung Cancer. N Engl J Med. 2017;377(20):1919-29.

26. Shaverdian N, Lisberg AE, Bornazyan K, Veruttipong D, Goldman JW, Formenti SC, Garon EB, Lee P. Previous radiotherapy and the clinical activity and toxicity of pembrolizumab in the treatment of non-small-cell lung cancer: a secondary analysis of the KEYNOTE-001 phase 1 trial. Lancet Oncol. 2017;18(7):895-903.

27. Chandra RA, Wilhite TJ, Balboni TA, Alexander BM, Spektor A, Ott PA, Ng AK, Hodi FS, Schoenfeld JD. A systematic evaluation of abscopal responses following radiotherapy in patients with metastatic melanoma treated with ipilimumab. Oncoimmunology. 2015:4(11):e1046028.

28. Sharabi AB, Lim M, DeWeese TL, Drake CG. Radiation and checkpoint blockade immunotherapy: radiosensitisation and potential mechanisms of synergy. Lancet Oncol. 2015;16(13):e498-509.

29. Ishiguro S, Sasazuki S, Inoue M, Kurahashi N, Iwasaki M, Tsugane S, Group JS. Effect of alcohol consumption, cigarette smoking and flushing response on esophageal cancer risk: a population-based cohort study (JPHC study). Cancer Lett. 2009;275(2):240-6.

30. Sakata K, Hoshiyama Y, Morioka S, Hashimoto T, Takeshita T, Tamakoshi A, Group JS. Smoking, alcohol drinking and esophageal cancer: findings from the JACC study. J Epidemiol. 2005;15(Suppl 2):S212-9.

31. Secretan B, Straif K, Baan R, Grosse Y, El Ghissassi F, Bouvard V, BenbrahimTallaa L, Guha N, Freeman C, Galichet L, et al. A review of human carcinogens--part E: tobacco, areca nut, alcohol, coal smoke, and salted fish. Lancet Oncol. 2009;10(11):1033-4.

32. Steevens J, Schouten LJ, Goldbohm RA, van den Brandt PA. Alcohol consumption, cigarette smoking and risk of subtypes of oesophageal and gastric cancer: a prospective cohort study. Gut. 2010;59(1):39-48.

33. Dale JE, Sebjornsen S, Leh S, Rosler C, Aaserud S, Moller B, Fluge O, Erichsen C, Nadipour S, Korner $\mathrm{H}$, et al. Multimodal therapy is feasible in elderly anal cancer patients. Acta Oncol. 2017;56(1):81-7. 
34. Cohen HJ, Smith D, Sun CL, Tew W, Mohile SG, Owusu C, Klepin HD, Gross CP, Lichtman SM, Gajra A, et al. Frailty as determined by a comprehensive geriatric assessment-derived deficit-accumulation index in older patients with cancer who receive chemotherapy. Cancer. 2016;122(24):3865-72.

35. Motzer RJ, Tannir NM, McDermott DF, Aren Frontera O, Melichar B, Choueiri TK, Plimack ER, Barthelemy P, Porta C, George S, et al. Nivolumab plus Ipilimumab versus Sunitinib in advanced renal-cell carcinoma. N Engl J Med. 2018;378(14):1277-90.

Ready to submit your research? Choose BMC and benefit from:

- fast, convenient online submission

- thorough peer review by experienced researchers in your field

- rapid publication on acceptance

- support for research data, including large and complex data types

- gold Open Access which fosters wider collaboration and increased citations

- maximum visibility for your research: over $100 \mathrm{M}$ website views per year

At $B M C$, research is always in progress.

Learn more biomedcentral.com/submissions 